\title{
Die Angst vor dem Sinnverlust nach der Diagnose Demenz. Eine Rekonstruktion unter Bezug auf Susan Wolf
}

\author{
Susanne Hiekel
}

Eingegangen: 12. April 2021 / Angenommen: 22. Juli 2021 / Online publiziert: 21. September 2021

(C) Der/die Autor(en) 2021

Zusammenfassung Verbunden mit dem drohenden Verlust von kognitiven Kapazitäten und der Veränderung der eigenen Persönlichkeit, ist zu vermuten, dass bei Demenzerkrankungen ein Sinnverlust vom Erkrankten befürchtet wird. Es ist Aufgabe dieses Papers aufzuklären, was es mit einer solch meist diffus empfundenen Angst auf sich hat. Dazu wird ein Deutungsangebot gemacht, das auf die Sinnkonzeption Susan Wolfs zurückgreift. Es wird gezeigt, dass es möglich ist, bis zu einem gewissen Grad der Demenz ein sinnvolles Leben zu führen - ein Leben, bei dem subjektiv Anziehendes und rational (bzw. objektiv) Wertvolles zusammengreifen müssen. Dies ist möglich, weil ein Mensch mit Demenz noch über entsprechende Fähigkeiten der Wertschätzung verfügt und eine Hilfsbedürftigkeit der Befähigung zu einem sinnvollen Leben nicht widerspricht. Bei einer sehr schweren Beeinträchtigung der kognitiven Kapazitäten wird es hingegen nicht mehr möglich sein, ein solches Leben zu leben; die solcherart erkrankte Person wird dies aber auch nicht mehr vermissen. Wenn das, was man mit der Demenzerkrankung zu verlieren befürchtet, durch das Sinnverständnis Wolfs getroffen ist, dann sollte man diese Überlegungen bei der Beurteilung einer empfundenen Angst berücksichtigen.

Schlüsselwörter Demenz $\cdot$ Angst $\cdot$ Lebenssinn $\cdot$ Susan Wolf

Dr. Susanne Hiekel $(\bowtie)$

Centrum für Bioethik, Westfälische Wilhelms-Universität Münster, Domplatz 23, 48143 Münster,

Deutschland

E-Mail: s.hiekel@uni-muenster.de 


\title{
The fear of losing meaning in life when dementia is diagnosed. A reconstruction with reference to Susan Wolf
}

\begin{abstract}
Definition of the problem Dementia is a disease which goes hand in hand with a decline of cognitive capabilities and changes in personality. Receiving such a grim diagnosis is quite likely accompanied by the fear that leading a meaningful life is no longer possible. Nevertheless, such fear will be rather diffuse, and clarifying what it could be about seems to be worthwhile. The aim of this paper is to elucidate this by drawing on Susan Wolf's influential conception of meaning in life.

Arguments It is shown that a meaningful life - up to certain stage of dementia-is possible: a life in which subjective attraction and rational (objective) attractiveness are joined together. This is because people with dementia have the ability to value in the respective way. The need for help is not undermining the possibility of leading such a life. In late stages, however, when the cognitive impairments are very severe, it will no longer be possible, but at this stage the demented person will not miss it either.

Conclusion When accessing the perceived fear, these considerations should be taken into account-provided that what one is afraid of fits to Wolf's understanding of meaning.
\end{abstract}

Keywords Dementia $\cdot$ Fear $\cdot$ Meaning in life $\cdot$ Susan Wolf

\section{Einleitung 1}

Ein Arztbesuch, der mit der Diagnosestellung einer Demenzerkrankung einhergeht, ist ein schockierendes Erlebnis, das nicht selten in tiefe existentielle Krisen führt. Der zukünftige Verlauf des Lebens wird in eine andere Richtung gelenkt, anders als man das vermutlich zuvor im Blick hatte. Die Diagnose wirft höchstwahrscheinlich Fragen für das zukünftige Leben auf, die man sich ohne eine solch einschneidende Mitteilung nicht stellen würde. Es kann sogar sein, dass Vorkehrungen für das zukünftige Leben im Sinne von Patientenverfügungen getroffen werden.

Ethische Probleme solcher Vorausverfügungen zu diskutieren, ist zwar ein wichtiger Bestandteil medizinethischer Debatten (vgl. z. B. Schöne-Seifert et al. 2019; Hallich 2011, 2019), wird aber in diesem Artikel keine Rolle spielen. Die Überlegungen hier schließen vielmehr an eine Wende in der empirischen Demenzforschung an. Neben einer Forschung entlang biomedizinischer Ansätze etablieren sich zunehmend personenzentrierte Forschungsansätze, die die subjektive Erfahrung der an einer Demenz Erkrankten in den Blick nehmen (vgl. Bender und Cheston 1997; O'Connor et al. 2007; Johannessen und Möller 2011). Ein Ergebnis dieser Forschung ist, dass die Demenz aus der subjektiven Perspektive als eine existentielle Bedrohung aufzufassen ist, die Angst hervorruft, mit der es umzugehen gilt (Che-

\footnotetext{
${ }^{1}$ Für hilfreiche Kommentare und Diskussionen zu früheren Versionen dieses Textes danke ich Oliver Hallich, Markus Rüther, Roland Kipke, Tim Rojek, Annette Brauer, Claudia Held und Anke Fehring.
} 
ston et al. 2015; Bjørkløf et al. 2019). Ein wesentlicher Aspekt bei der Wahl einer geeigneten Bewältigungsstrategie ${ }^{2}$ ist es, sich darüber klar zu werden, vor was man genau Angst hat und ob die Angst berechtigt ist.

Menschen, die die Diagnose Demenz gestellt bekommen haben, sind mit einer ganzen Reihe von zu erwartenden bedrohlichen Veränderungen in ihrem Leben konfrontiert. Ein Konstituens der existentiellen Bedrohung durch Demenz kann es sein, dass man befürchtet, ein sinnvolles Leben nicht mehr führen zu können (vgl. Holst und Hallberg 2003; Cheston et al. 2015). Dieses Phänomen - die Angst vor dem drohenden Sinnverlust - soll im Folgenden näher analysiert werden. Verbinden Menschen mit Demenz Angst mit dem drohenden Sinnverlust, so ist es wichtig für sie selbst, aber auch für Ärzte, Pflegende und Angehörige zu klären, was es mit dieser Angst auf sich hat.

So wahrscheinlich es ist, dass nach der Diagnosestellung Demenz Überlegungen zur Sinnhaftigkeit des späteren Lebens eine Rolle spielen werden, so unklar ist zunächst, was mit ,Sinnhaftigkeit“ überhaupt ausgedrückt wird. Ein Leben als sinnlos zu qualifizieren, ist alltagssprachlich eine vage Aussage, die vielerlei Bedeutungen haben kann. Werden Menschen mit Demenz danach gefragt, wie sie Sinnhaftigkeit erfahren, so benennen sie unterschiedliche Aspekte, welche Dewitte et al. (2021, S. 1) metaphorisch mit ,continuing to participate in the dance of life as oneself“ zusammengefasst haben. Hier soll ein philosophisches Deutungsangebot für bestimmte Aspekte, die unter diesem Bild zusammengefasst werden, unter Rückgriff auf die Sinnkonzeption Susan Wolfs (Wolf 1997, 2010c, 2015, 2016; vgl. Hiekel 2020) gemacht werden. ${ }^{3}$

Der Ansatz von Wolf wird gewählt, weil Wolf selbst Situationen der Verzweiflung oder der Epiphanie als paradigmatische Situation benennt, in denen man mit sich mit der Sinnhaftigkeit des Lebens auseinandersetzt. Das Erleben der Demenz kann beiden Situationen zugeordnet werden (Holst und Hallberg 2003; Bender und Cheston 1997). Zudem passt die Perspektive, aus der heraus Wolf die Sinnhaftigkeit im Leben analysiert, zum personenzentrierten Demenzforschungsansatz, da ihre Analyse ebenfalls von der Perspektive der Personen ihren Ausgang nimmt, die sich die Sinnfrage stellen. Wesentlich ist aber, dass Wolfs Ansatz mit den empirischen Forschungsergebnissen harmoniert, die darauf verweisen, dass Menschen mit Demenz ein sinnvolles Leben mit einer Beschäftigung in sinnvollen Tätigkeiten verbinden (vgl. O'Connor et al. 2007) bzw. dass sie einen Zweck erfüllen, nützlich sein, etwas Wertvolles beitragen wollen (Dewitte et al. 2021). Eine Beschäftigung in sinnvollen bzw. wertvollen Projekten oder Tätigkeiten ist wiederum ein essenzieller Bestandteil der wolfschen Konzeption.

\footnotetext{
2 Mögliche Bewältigungsstrategien für Menschen, die sich mit der Demenzerkrankung konfrontiert sehen, fassen Bjørkløf et al. (2019, S. 16) in vier Kategorien zusammen: „,(1) Keep going and holding on to life as usual; (2) Adapting and adjusting to the demands from the situation; (3) Accepting the situation; and (4) Avoiding the situation [...]“.

3 Es existieren in der aktuellen philosophischen Debatte mehrere Angebote, wie ein Sinn im Leben verstanden werden kann (vgl. Rüther und Muders 2014; Rüther 2021a, b) und auch die Bezüge, die sich innerhalb der Medizinethik zur Sinnthematik herstellen lassen, sind vielfältig, wie das Editorial zum Themenheft „Die Sinnfrage in der Medizinethik“ aufzeigt (vgl. Kipke und Rüther 2021).
} 
Es wird hier gezeigt, dass - bis zu einem gewissen Grad der Demenz - ein im wolfschen Sinne sinnhaftes Leben mit Demenz möglich ist. Im Fall einer schweren Demenz hingegen ist ein solch sinnhaftes Leben nicht mehr möglich. Tröstlich mag sein, dass man die Verunmöglichung dieses Sinns im Stadium der schweren Demenz aber auch nicht vermissen wird. Hier ist allerdings darauf hinzuweisen, dass der Ausdruck ,Sinn“ notorisch mehrdeutig ist. Das Urteil, dass ein Leben nicht sinnhaft ist oder sein kann, kann vielerlei bedeuten. Es ist zu betonen, dass das Urteil gänzlich auf das wolfsche Sinnverständnis bezogen ist. Es soll auf keinen Fall so verstanden werden, dass damit Wertlosigkeit, Überflüssigkeit o.ä. attestiert wäre.

\section{Demenzerkrankungen}

Bei Demenzerkrankungen handelt es sich um degenerative Gehirnerkrankungen, die mit sukzessive zunehmenden kognitiven, funktionalen und sozial-interaktiven Einbußen einhergehen, ohne dass es zu einem Verlust an einer Tiefe von Erlebnis- und Gefühlsmomenten kommen muss. Der symptomatische Verlauf der Erkrankung ist progredient und wird üblicherweise in drei Stadien unterteilt: die leichte, die mittlere und die schwere Demenz. In der leichten Demenz erleben die Menschen, die daran erkrankt sind, kognitive Defizite in leichter Ausprägung. In dieser Phase sind die Patient*innen noch recht selbstständig und sie nehmen die Veränderungen, denen sie unterworfen sind, häufig wahr. In der mittelschweren Demenzphase werden die kognitiven Einbußen stärker. Es kommt zu auffälligen Gedächtnis- und Orientierungsstörungen. Diese Veränderungen werden teils wahrgenommen, teils aber auch nicht. Die Patient*innen sind zunehmend auf die Hilfe anderer angewiesen, um die täglichen Dinge des Lebens zu verrichten. In der Phase der schweren Demenz sind Erkrankte dann vollständig auf die Hilfe anderer angewiesen. Die Person, die sich im Finalstadium der Demenzerkrankung befindet, hat in Bezug auf Vorlieben, Verhalten, Charaktereigenschaften und kognitive Fähigkeiten kaum noch Ähnlichkeiten mit der Person vor der Erkrankung, obwohl natürlich eine körperliche Kontinuität besteht.

Es erscheint als ein tragisches Moment der Demenzerkrankung, dass man miterlebt, wie die kognitiven Fähigkeiten schwinden, mit deren Hilfe man zuvor das eigene Leben gestaltet hat, und wie sich die eigene Persönlichkeit verändert. Richard Taylor, ein Psychologieprofessor, der mit 58 Jahren die Diagnose „Demenz, vermutlich vom Alzheimer Typ“ bekam, hat als Alzheimer-Aktivist sein Erleben der Erkrankung dokumentiert. Mit Hilfe von digitaler Sprach- und Erinnerungstechnologie hat er es geschafft zu erläutern, wie er den Übergang von der zweiten zur dritten Stufe erlebt (Taylor 2015b). Sehr eindrücklich beschreibt er diesen Zustand, der sich dadurch auszeichnet, dass sich die Wahrnehmung verschiebt, das Verständnis dessen, was wahrgenommen wird, sich verändert und dass auch das Selbstverständnis und die eigene Identität in Frage gestellt werden. Taylor sagte z. B. in einem SpiegelInterview: „Man verwandelt sich nach und nach in einen Menschen, den man noch nicht kennt, und der, den man kannte, verschwindet" (Lakotta 2010).

Leidvolle Veränderungen, die mit der Demenzerkrankung einhergehen, können die Frage nach der Sinnhaftigkeit eines solchen Lebens provozieren (vgl. Wolf 1997, 
S. 6f; Diehl 2009). Sinnhaftigkeit kann ein Teil dessen sein, was man mit der eigenen Persönlichkeit in Verbindung bringt. Man möchte eine Person sein, deren Leben sinnvoll ist, und fragt sich, ob bei einer Änderung der eigenen Persönlichkeit im Demenzfall auch dieser Aspekt zu schwinden droht.

Bevor die Angst vor einem Sinnverlust genauer analysiert wird, lohnt es, sich zunächst Gedanken darüber zu machen, was es heißen soll, dass man eine Veränderung der Persönlichkeit bewertet.

\section{Die Bewertung einer Persönlichkeitsveränderung}

Durch die Demenz wird die personale Identität im Sinne der Persönlichkeit bedroht (vgl. Lesser 2006; McMillan 2006; Radden und Fordyce 2006). ${ }^{4}$ Die Persönlichkeit droht sich in einer für den Betroffenen/die Betroffene nicht akzeptablen Weise zu verändern. Was es allgemein mit der Bewertung einer Persönlichkeitsveränderung auf sich hat, wird hier anhand der Konzeption der praktischen Identität Christine Korsgaards erläutert.

Jeder kennt das Phänomen, dass man in Bezug auf die eigene Person Bewertungen vornimmt. Eine solche Beurteilung des eigenen Selbst unterscheidet Menschen von anderen nicht-menschlichen Tieren: Wir verfügen über ein reflexives Bewusstsein, das uns befähigt, auf uns selbst beschreibend, beurteilend und vorschreibend Bezug zu nehmen (vgl. Korsgaard 2018, S. 46). Wir verfügen über eine normative oder evaluative Selbstkonzeption. Deshalb betrachten wir es als unsere Angelegenheit zu bestimmen, wer oder was wir sein wollen. Die eigene Persönlichkeit ist daher nicht etwas, das einfach gegeben ist. Wir schaffen unsere Identität dadurch, dass wir uns mit dem selbst Wertgeschätzten identifizieren. Korsgaard versteht unter der eigenen Identität, die jemanden dazu veranlasst, in bestimmter Weise zu agieren, ,a description under which you value yourself, a description under which you find your life to be worth living and your actions worth undertaking“ (Korsgaard 2014, S. 101).

Die Beschreibungen, die z. B. auch auf das soziale oder berufliche Leben bezogen sein können (bspw. Freund*innen, Ehepartner*innen etc. sein, bzw. Lehrer*innen, Wissenschaftler*innen, Kindergärtner*innen etc.), leiten durch die eigene Wertschätzung das eigene Leben insbesondere langfristig an. Das aktuale Selbst legt durch diese Wertschätzungen fest, wie das zukünftige Selbst handeln soll. Was bedeutet nun die Bewertung einer Persönlichkeitsveränderung unter diesem korsgaardschen

\footnotetext{
${ }^{4}$ Die Frage nach der Identität der Person kann nach Quante (2013) in (mindestens) vier Problemfelder unterteilt werden. Das sind 1. das Problem der Personalitätsbedingungen, 2. das Einheitsproblem der Person, 3. das Persistenzproblem der Person und 4. das Personalitätsstruktur-Problem. Die Ausführungen in diesem Text sind dem letztgenannten Problemfeld zuzuordnen, bei dem es um das evaluative bzw. normative Selbstverständnis von Subjekten geht und darum, welche Struktur grundlegend dafür ist, ein Leben als Person nicht nur zu haben, sondern auch zu führen (vgl. Rojek 2020, S. 151). Identität ist in diesem Kontext nicht als Relation zu denken, sondern als ein evaluatives oder normatives Konzept des Selbstverständnisses oder Selbstbildes einer Person (vgl. Quante 2007, S. 11). In diesem Sinne ist auch die Rede vom „Selbst“ zu verstehen. Damit steht weder eine metaphysisch aufgeladene Idee eines wahren Selbst noch eine verdinglichende Ontologisierung in Verbindung.
} 
Verständnis? Das kann man sich vor Augen führen, indem man sich eine Bewertung der Persönlichkeitsveränderung vergegenwärtigt. Sowohl retrospektive als auch prospektive Bewertungen sind möglich, wobei letztere insbesondere für den Demenzkontext von Relevanz sind.

Unter einer prospektiven Persönlichkeitsveränderung kann man eine antizipierte Veränderung der eigenen Persönlichkeit verstehen. Ein Fünfzigjähriger kann sich vorstellen, wie er mit neunzig Jahren wohl sein wird und was er unter dieser Beschreibung wertschätzen wird. Vielleicht werden körperliche Einschränkungen ein Sicherheitsbedürfnis stark werden lassen, so dass eine Beschreibung des eigenen Selbst, das sich mit Hilfe eines Rollators fortbewegt, für den Neunzigjährigen etwas sein kann, das er begrüßt oder zumindest akzeptiert. Die Beschreibung des eigenen fünfzigjährigen Selbst, das sich an einem Rollator fortbewegt, wird hingegen etwas sein, das vermutlich mit Ablehnung verbunden sein wird. Der Fünfzigjährige kann sich nun überlegen, wie er eine solche Veränderung im Spektrum der Wertschätzung dessen, wie er sich beschreibt, bewertet. Allein die Auseinandersetzung mit dieser möglichen Veränderung kann dazu führen, dass sich die Beschreibung seiner selbst bzw. die Bewertung ändert. Sah man sich vor der Auseinandersetzung nur als der Fünfzigjährige zum jetzigen Zeitpunkt, sieht man sich in der Auseinandersetzung mit diesen Überlegungen als Fünfzigjähriger, der irgendwann einmal 90 Jahre alt sein wird. Es kann dazu kommen, dass man manche Veränderungen bedauern wird, manche begrüßen, aber auch, dass einen manche unberührt lassen. In der Auseinandersetzung mit möglichen Veränderungen seiner selbst in der Zukunft kann das zur Folge haben, dass diese Überlegungen dazu führen, dass sich die praktische Identität des Fünfzigjährigen verändert, weil bestimmte Veränderungen in der Zukunft ihn vielleicht unberührt lassen, ihm wünschenswerter als andere erscheinen oder aber auch manche als nicht akzeptabel befunden werden.

Was haben diese Überlegungen mit der Angst vor einem Sinnverlust zu tun? Wer sich Gedanken darüber macht, was das eigene Leben zu einem sinnvollen gemacht hat, macht oder machen wird, und eine Antwort darauf gefunden hat, der gibt damit eine bestimmte Beschreibung seiner selbst, die er/sie wertschätzt und die für das eigene Leben normativ leitend ist. Die Angst vor einem Sinnverlust ist dann die Angst davor, etwas zu verlieren, das man in der Beschreibung seiner selbst wertschätzt und das einem das Leben lebenswert erscheinen lässt. Man erhält die Diagnose „Demenz“ und fragt sich, wie man sein Leben, sein eigenes Selbst beurteilt, wenn man zu einem sinnvollen Leben evtl. nicht mehr fähig ist. Es bleibt allerdings zu klären, was genau es heißen soll, sich um einen Sinn im Leben Sorgen zu machen. Deshalb wird im Folgenden eine Konzeption sinnvollen Lebens vorgestellt.

\section{Einen Sinn im Leben haben - Die Konzeption Susan Wolfs}

Susan Wolf orientiert sich in ihrer Konzeption sinnvollen Lebens an unserem alltäglichen Sprachgebrauch. Sie erläutert, was ein sinnvolles Leben ausmacht, indem sie Negativbeispiele anführt - also Beispiele, denen wir den Ausdruck ,,sinnvolles Leben“ nicht zuschreiben würden. Man würde ein Leben, das die Merkmale 
von Aktivität und Engagement nicht aufweist (The Blob) oder in denen man sich in Projekten engagiert, die - in einem nicht genau spezifizierten Sinne - objektiv nicht wertvoll sind (The Useless) nicht als sinnvoll bezeichnen (vgl. Wolf 2015). ${ }^{5}$ Es sind also aktives Engagement und die objektive Werthaftigkeit von Aktivitäten oder Projekten, die ein Leben sinnvoll machen. In dieser Hinsicht macht Wolf zusätzlich geltend, dass beide Elemente auf bestimmte und geeignete Weise miteinander verbunden sein müssen, um von einem sinnvollen Leben sprechen zu können. Subjektives und Objektives müssen aufeinander bezogen sein, damit man von Sinn im Leben sprechen kann, denn ,,meaning arises when subjective attraction meets objective attractiveness" (Wolf 2010a, S. 9). Aus diesem Grund bezeichnet sie ihre Konzeption auch als Fitting Fulfillment View. Ein sinnvolles Leben ist dadurch ausgezeichnet, dass in der subjektiven Erfüllung erkannt wird, dass das, was erfüllend, auch objektiv wertvoll ist: „The thought is that if one tries it, one will like it, and one will like it in part because of one's recognition that one is engaged with a person or an object or an activity that is independently valuable“ (Wolf 2010a, S. 22).

Für den Fitting Fulfillment View Wolfs ist eine objektive Werthaftigkeit konstitutiv. Dieses Element der wolfschen Sinnhaftigkeit ist jedoch kritisch zu sehen, da Wolf erstens nicht erläutert, was unter objektiver Werthaftigkeit zu verstehen ist, und zweitens eine objektive Werthaftigkeit unter Verdacht steht, metaphysisch aufgeladen und rein intuitionistisch begründbar zu sein (vgl. zur Kritik an objektiver Werthaftigkeit Mackie 1977). Da die Gesamtkonzeption Wolfs aber durchaus mit einer weniger metaphysisch belasteten rationalen Werthaftigkeit kompatibel ist, schlage ich vor, dass für die weitere Analyse objektive Werthaftigkeit durch rationale Werthaftigkeit ersetzt wird (vgl. Hiekel 2018). Etwas ist rational wertvoll, wenn es wohlbegründet ist, es für gut zu befinden, d.h., dass die Wertschätzung auf einer intersubjektiven Basis beruht. Man kann also sagen: ,[M]eaning arises when subjective attraction meets rational attractiveness" (Hiekel 2018, S. 70).

Manche Projekte können mit großer Sicherheit von einem rationalen Standpunkt aus als wertvoll verstanden werden. Das sind solche, die Wolf selbst auch als paradigmatische Beispiele anführen würde, wie beispielsweise sein Leben der Wissenschaft zu widmen (z. B. wie Charles Darwin), der Kunst (z. B. wie Frida Kahlo), der Musik (z.B. wie Nina Simone) oder der Medizin (z. B. wie Robert Koch). Bei manchen ist man sich sicher, dass sie es nicht sind: bei Projekten, die sozusagen einen Negativwert besitzen (z. B. Menschen oder Tiere quälen) oder sinnfrei sind (z. B. Grashalme zählen). Es wird aber auch sicherlich Projekte geben, bei denen man sich erst noch genau überlegen müsste, ob es gute Gründe gibt, diese zu verfolgen oder nicht.

Wie hängt diese Überlegung nun mit denen zusammen, die zuvor in Bezug auf die Persönlichkeit angestellt wurden? Die Sinnhaftigkeit des eigenen Lebens - das Verfolgen von Projekten, die zugleich subjektiv wie auch rational wertvoll sind ist etwas, das in der Beschreibung des eigenen Selbst wertgeschätzt werden kann

\footnotetext{
5 Wolf diskutiert auch noch den Fall von „The Bankrupt“, bei dem sich jemand aktiv in einem Projekt von Wert engagiert, sich mit dem, was er tut, völlig identifiziert, aber das Projekt nicht zum Erfolg führen kann. Das damit eingeführte Erfolgskriterium für die Sinnhaftigkeit ist allerdings kritisch zu sehen (vgl. Koethe 2010).
} 
und das einem das Leben lebenswert erscheinen lassen kann. ${ }^{6}$ Das kann explizit der Fall sein, kann einem aber auch erst deutlich werden, wenn die Diagnose „Demenz“ gestellt wird. Man erkennt, dass man bestimmte Beschreibungen seiner selbst in Bezug auf verfolgte oder zukünftige Projekte wertschätzt und befürchtet, dass eine Verfolgung derselben nicht mehr möglich ist. Wenn das der Fall ist, dann ist oder wird die Sinnhaftigkeit des eigenen Lebens als faktischer oder potenzieller Teil der praktischen Identität, als Teil der eigenen Persönlichkeit, durch die Demenzerkrankung in Frage gestellt. Da dies unfreiwillig - sozusagen aufgezwungen - passiert, kann das als starke Bedrohung für die eigene Person empfunden werden.

\section{Was passiert mit dem Sinn in der Demenz?}

Der Mensch, der bei seinem Arztbesuch mitgeteilt bekommt, dass er eine Demenzerkrankung hat, und befürchtet, dass die Sinnhaftigkeit seines Lebens bedroht ist, befürchtet also nach dem Ansatz von Susan Wolf, dass es ihm nicht (mehr) möglich sein wird, ein Leben zu führen, in dem er Projekte verfolgt, die ihn subjektiv erfüllen und die gleichzeitig rational wertvoll sind. Das wäre die philosophische Deutung für Äußerungen, die Menschen mit Demenz tätigen, wenn sie befürchten, keine sinnvollen oder wertvolle Tätigkeiten mehr ausführen zu können. Obwohl Richard Taylor - der Alzheimer-Aktivist - diese Überlegungen m. W. nicht explizit angestellt hat, könnte man sich vorstellen, dass er die Sinnhaftigkeit seines Lebens in Gesundheit z. B. mit seiner Selbstständigkeit, der liebevollen Beziehung zu seiner Frau und seinen Kindern und mit seiner beruflichen Tätigkeit als Psychologieprofessor in Verbindung bringt. Sie machen einen Teil seiner Persönlichkeit aus und für diese Aspekte gilt (höchst wahrscheinlich), dass sie ihn subjektiv erfüllen und dass es gute Gründe gibt, diese sozusagen als Projekte zu verfolgen. Diese Aspekte seiner eigenen Persönlichkeit drohen sich durch die Demenzerkrankung stark zu verändern bzw. verloren zu gehen. Metaphorisch spricht er davon, dass solche Aspekte zu dunklen Räumen seines Gedächtnisses werden (vgl. Taylor 2015a).

Ist es aber so, dass das Leben von Menschen mit Demenz notwendigerweise die Sinnhaftigkeit durch den Prozess der Demenzerkrankung verliert oder besteht die Möglichkeit, dass sich die Sinnhaftigkeit verändert?

\section{Die Sinnhaftigkeit eines Lebens mit (leichter bis mittelschwerer) Demenz}

Die Antwort ist, dass auch unter einer Manifestation der Demenzerkrankung ein sinnvolles Leben möglich ist, weil der Demenzerkrankte immer noch Dinge in seinem Leben für wertvoll erachten kann, die subjektiv erfüllend und die rational wertvoll sind. Man könnte allerdings einwenden, dass das nicht möglich ist, da der

\footnotetext{
${ }^{6}$ Das heißt nicht, dass z. B. The Blob nicht auch sein Leben wertschätzen kann. Ein sinnhaftes Leben zu führen, ist nicht notwendig dafür, dass man aus der Binnenperspektive sein Leben für lebenswert einschätzt. Das heißt auch nicht, dass man dem Leben von The Blob den Lebenswert absprechen würde. Da aber Sinnhaftigkeit - neben Glück oder Moralität - als eine Dimension des guten Lebens aufgefasst werden kann, wäre es rational, einen Verlust von Sinn zu bedauern (vgl. Wolf 2016; Muders 2018; Kipke 2018; kritisch dazu allerdings vgl. Halbig 2018).
} 
Demenzerkrankte die Fähigkeit verloren hat, selbstbestimmt etwas - im Sinne eines kritischen Interesses - wertzuschätzen, und nicht mehr fähig ist, etwas, das rational wertvoll ist, zu verfolgen. Dieser Einwand kann allerdings zurückgewiesen werden, denn an Demenz erkrankte Personen können durchaus noch etwas selbstbestimmt wertschätzen, und es gibt auch Beispiele dafür, dass Menschen mit Demenz Projekte verfolgen, für die es gute Gründe gibt, sie wertzuschätzen - wie z. B. die Aktivitäten Richard Taylors als Alzheimer-Aktivist zeigen. Das Verfolgen solcher Projekte bedarf nur der Hilfe. Dieses Angewiesensein auf Hilfe konfligiert dabei nicht, wie man denken könnte, mit dem Gedanken einer autonomen Wertschätzung. Hilfsbedürftigkeit und Autonomie sind miteinander vereinbar. Für diese Einschätzung greife ich auf eine Argumentation von Agnieszka Jaworska (1999) zurück. Jaworska wendet sich in ihrem Artikel gegen die einflussreiche Position Roland Dworkins in der Debatte um Patientenverfügungen. Die Stoßrichtung der Argumentation Jaworskas geht also in eine etwas andere Richtung, aber man kann die grundlegenden Gedanken für die Überlegungen zum Sinnkontext fruchtbar machen.

Zunächst die Position von Jaworskas Opponenten: Dworkin geht davon aus, dass der Wille einer (noch) kompetenten Person ausschlaggebend dafür ist, wie später mit der kognitiv beeinträchtigten Person mit Demenz umgegangen werden sollte. Warum geht er davon aus? Er denkt, dass die Person, die die Patientenverfügung erlässt, über die Fähigkeit der autonomen Entscheidung verfügt, und dass diese Entscheidung daher zu respektieren ist. Sie verfügt über sogenannte kritische und erlebnisbezogene Interessen, wohingegen die Person mit manifesten Demenzsymptomen nur noch über erlebnisbezogene Interessen verfügt. ${ }^{7}$ Die kognitiv beeinträchtigte Person verfügt deshalb nicht mehr über die Fähigkeit zur Autonomie, und aus diesem Grund ist die Entscheidung der kognitiv kompetenten Person autoritativ. Mit den kritischen Interessen bringt die Person zum Ausdruck, wie sie ihr Leben entwerfen möchte, während die erlebnisbezogenen Interessen bloß dem Moment verhaftet sind. Denkt man diese Argumentationslinie weiter, so hieße das - übertragen auf den Kontext der Sinnfrage -, dass nur die noch kompetente Person ein sinnhaftes Leben führen kann, weil nur sie über die Fähigkeit verfügt, kritische Interessen zu haben. Nur sie ist fähig zu entscheiden, in welcher Art und Weise sie ihr Leben zu führen wünscht. Nur sie ist zu dieser Art Selbstbestimmung fähig.

Der Sichtweise Dworkins hält Jaworska allerdings entgegen, dass auch die kognitiv eingeschränkte Person die Fähigkeit zur Wertschätzung von etwas besitzt und damit auch dazu, kritische Interessen zu haben. Daher kann man auch sie als autonom bezeichnen. Während Dworkin denkt, dass kritische Interessen sich auf das Leben als Ganzes - ,a whole life, a past joined to a future“ (Dworkin 1993a, S. 230) - beziehen müssen, meint Jaworska, dass kritische Interessen etwas weniger anspruchsvoll verstanden werden sollten. Dworkin verbindet nämlich zwei Aspekte miteinander, die nicht notwendig miteinander verknüpft sind: eine Überzeugung da-

\footnotetext{
7 Erlebnisbezogene Interessen (experiential interests) sind solche, die auf das bezogen sind, was wir erleben, wenn wir bestimmte Dinge tun. Man hat Interesse an bestimmten Tätigkeiten oder Gegenständen, weil sie mit positiven Empfindungen verbunden sind. Kritische Interessen (critical interests) sind persönliche Interessen, die auf etwas gerichtet sind, von dem man überzeugt ist, dass es wertvoll ist und das unabhängig davon, welche Erfahrungen aus der Erfüllung dieser Interessen für einen selbst resultieren (vgl. Dworkin 1993b, S. 201ff).
} 
von haben zu können, wie man sein Leben führen möchte, und ein Verständnis vom eigenen Leben im Ganzen haben zu können. Jaworska argumentiert dafür, dass kritische Interessen lediglich mit der Fähigkeit der Wertschätzung verbunden sind und dass in diesem Zusammenhang ein Bezug zum Leben als Ganzes nicht notwendig ist.

Für die Annahme, dass in bestimmten Situationen kritische Interessen vor den bloß erlebnisbezogenen Interessen vorrangig zu berücksichtigen sind, benötigt es nämlich üblicherweise keinen Bezug auf das Leben als Ganzes. Jaworska bringt dafür das Beispiel eines Vaters, der dem kritischen Interesse, zum Wohle seiner Kinder zu handeln, gegenüber dem Interesse, selbst optimale Erfahrungen zu machen, den Vorrang gibt, ohne dabei auf sein Leben als Ganzes Bezug zu nehmen (Jaworska 1999, S. 117). Ähnliches spiegelt sich auch z. B. in der medizinischen Praxis im Kontext der informierten Zustimmung von Patienten. Auch dort ist die Beachtung der informierten Zustimmung von Patienten Ausdruck des Respekts vor der Autonomie, ohne dass dabei eine Reflexion auf das Leben als Ganzes gefordert würde. Die kritischen Interessen werden deshalb als so wichtig erachtet, weil sie das repräsentieren, was eine Person für das eigene Leben wertschätzt. Dafür ist es nicht notwendig, dass die Person eine Vorstellung von einem Entwurf ihres Lebens im Ganzen besitzt (vgl. Jaworska 1999, S. 116).

Dass man etwas in seinem Leben wertschätzt (to value - im Gegensatz dazu, etwas bloß zu wünschen (to merely desire)), heißt nach Jaworska:

[T] he person thinks she is correct in wanting what she wants; achieving what she wants is tied up with her sense of self-worth; and the importance of achieving what she wants is, for her, independent of her own experience. Nothing here suggests that valuing would require a grasp of the narrative of one's whole life. ${ }^{8}$ (Jaworska 1999, S. 116)

Und gerade letzteres Merkmal ist es, das Demenzerkrankte zu verlieren drohen (a grasp of the narrative of one's whole life), nicht aber die Fähigkeit, etwas für ihr Leben wertzuschätzen. An Demenz Erkrankte können sich immer noch erfüllt von etwas fühlen und das als Teil ihrer evaluativen Selbstkonzeption befürworten. Patienten, die Jaworska beschreibt, und auch der Alzheimer-Aktivist Richard Taylor können dafür als Beispiele herangezogen werden.

So berichtet Jaworska von Patienten, die an einer Alzheimer-Studie teilgenommen haben. Eine Patientin - Mrs. D. - war bereits fünf Jahre an Alzheimer erkrankt und ihr Zustand entsprach zum Zeitpunkt der Studie dem einer mittelschweren Demenz. Sie war zeitlich und örtlich desorientiert und hatte Schwierigkeiten, neue Erinnerungen zu formen. Man kann daher sagen, dass sie kein Konzept von ihrem Leben als Ganzes mehr besaß. Dennoch verfügte sie über die Fähigkeit, etwas für ihr Leben wertzuschätzen. Sie begründete beispielweise ihre Teilnahme an der Alzheimer-Studie damit, dass sie dadurch anderen Menschen helfen könne. Darüber hinaus bemühte sie sich auch immer mit einer ähnlichen Begründung, bei anderen Patienten eine gute Stimmung zu verbreiten (Jaworska 1999, S. 118). Mrs. D. erfüllt

\footnotetext{
${ }^{8}$ Diese Sichtweise dessen, was es heißt, etwas wertzuschätzen, entwickelt Jaworska in enger Verbindung zum korsgaardschen Verständnis der praktischen Identität in ihrer Dissertationsschrift (Jaworska 1997).
} 
daher nach Jaworska die Kriterien für die Fähigkeit der Wertschätzung, die oben genannt wurden. Mrs. D. denkt, dass es richtig ist, anderen Menschen helfen zu wollen. Ihre Bereitschaft zu helfen steht in Verbindung zu ihrem Selbstwertgefühl und das Objekt ihrer Wertschätzung ist nicht das eigene Erleben von etwas, sondern etwas davon Unabhängiges.

Auch Richard Taylor kann diese Kriterien erfüllen. Er sieht es als sein Projekt an, das Leben mit Alzheimer zu dokumentieren, damit das anderen Alzheimer-Patienten zugutekommt. Er schreibt selbst:

Your writings will not end up in high school literature classes or on poetry books, but they have potentially profound value to all who are living today just like yesterday, and tomorrow just like today. We have the opportunity to offer others the opportunity to learn about themselves based on more than simply their own experience. (Taylor 2015c, S. 187)

Mir scheint Richard Taylor ein paradigmatischer Fall für jemanden zu sein, der ein sinnhaftes Leben führt. Man kann ihn sehr gut in die Reihe derjenigen Personen integrieren, die üblicherweise als typische Beispiele sinnhaften Lebens angeführt werden. Taylor ist subjektiv erfüllt von dem, was er tut, und weiß auch, dass es dafür gute Gründe gibt.

Der Unterschied, den man hier im Gegensatz zu gesunden Personen allerdings machen könnte, wäre der, dass Richard Taylor oder Mrs. D. auf Hilfe angewiesen sind, um sich den Projekten zu widmen, die sie für ihr Leben wertschätzen und die auch in rationaler Weise wertzuschätzen sind. Sie brauchen Hilfe in der Form von Personen, die ihnen ermöglichen, sich zeitlich und örtlich zu orientieren. Richard Taylor benötigt auch technische Unterstützung, um sein Projekt voranzutreiben. Kann man unter diesen Umständen immer noch sagen, dass die Projekte tatsächlich die Projekte dieser Personen sind - Projekte, in denen sie selbst aktiv engagiert sind? Ich denke ja, das kann man, und zwar aus (mindestens) zwei Gründen: Erstens ist kaum jemand imstande, Lebensprojekte ohne Hilfe oder Hilfsmittel voranzutreiben, und zweitens ist es begrifflich nicht in der Bestimmung eines selbstbestimmten Lebens enthalten, dass Hilfsmittel oder Hilfe keine Rolle spielen dürfen.

Es ist klarerweise der Fall, dass die meisten Lebensprojekte, in denen man sich engagiert, bestimmte Hilfsmittel benötigen. Richard Taylor benötigt für sein Projekt nur mehr technisches Equipment als üblich. Allerdings stellt dieses Equipment für Taylor sicher, dass er sich an die Dinge selbst, die er zu verfolgen wünscht, und an die damit verbundenen Informationen erinnert. Ist das nicht etwas anderes, weil Taylors Hilfsmittel seine mentalen Fähigkeiten unterstützen? Das sind in diesem Sinne tatsächlich andere Hilfsmittel, aber sie schließen nicht aus, dass sich Taylor immer noch selbst aktiv engagiert. Man kann das technische Equipment als Teil von erweiterten kognitiven Prozessen Taylors ansehen (vgl. Clark und Chalmers 1998).

Allerdings benötigen Personen mit Demenz bei der Verfolgung dessen, was sie wertschätzen, auch Hilfe durch andere Personen, die ihnen dies überhaupt erst ermöglichen. Wenn die Familie von Richard Taylor die Hilfsmittel etc. nicht bereitstellen und warten würde, dann könnte er das Erleben seiner Demenzerkrankung aus eigener Perspektive nicht dokumentieren. Er kann sich nicht selbst darum kümmern, dass die geeigneten Mittel zur Verfolgung seiner Zwecke vorhanden und zugäng- 
lich sind. Erst in der Beziehung zu anderen stehend, ist es der Person mit Demenz möglich, das zu tun oder so zu sein, wie sie es wertschätzt.

Aber auch diese Tatsache spricht nicht dagegen, dass auch Menschen mit Demenz ein sinnhaftes Leben führen können. Dass sie Beistand brauchen, um sich erfüllt zu fühlen und Dinge zu verfolgen, die rational wertvoll sind, ist kein Grund dafür, diesem Leben in einem gewissen Sinne Selbstbestimmtheit oder Sinnhaftigkeit abzusprechen. Auch dies ist ein Aspekt, auf den Jaworska in Bezug auf die Fähigkeit zur Autonomie aufmerksam macht. Mit ihrem Verständnis von Autonomie, das wesentlich mit der Fähigkeit der Wertschätzung (capacity to value) einhergeht, kann man Personen mit Demenz bis in fortgeschrittene Stadien des Krankheitsverlaufs Autonomie zusprechen. Was sie allerdings nicht mehr vollumfänglich oder auch nur noch rudimentär besitzen, ist die Fähigkeit, entsprechend der Ziele geeignete Mittel zu ergreifen (vgl. Jaworska 1999, S. 126).

Würde man das Konzept der Selbstbestimmtheit streng an die Fähigkeit binden, in vollständig eigenständiger Weise geeignete Mittel zur Erreichung dessen ergreifen zu können, was man anstrebte, so würde kaum noch jemand die Anforderungen an Autonomie erfüllen können. Wer kann schon völlig auf sich gestellt das verfolgen, was er oder sie wertschätzt? Autonomie muss aber auch nicht im Sinne einer völligen Unabhängigkeit von anderen verstanden werden. Menschen sind eingebettet in soziale Strukturen und Beziehungen und zum Teil auch angewiesen auf andere, wenn es darum geht, das eigene Leben zu gestalten. Das ist kompatibel damit, dass sie autonom sind. Selbstbestimmtheit geht nicht zwingend mit völliger Unabhängigkeit einher (vgl. Mackenzie und Stoljar 2000). Im Falle der Personen mit Demenz geht die Abhängigkeit zugegebenermaßen weiter als das bei Personen der Fall ist, die nicht kognitiv beeinträchtigt sind. Die betreuende Person muss nicht nur dabei behilflich sein, das zu erreichen, was jemand für gut befindet, sondern muss auch unter Umständen identifizieren, was der Betreute für gut befindet. Ab einem bestimmten Grad der Erkrankung sind an Demenz Erkrankte abhängige Akteure (dependent agents), deren Präferenzen, Interessen und Wertsetzungen in Kooperation mit Hilfspersonen verwirklicht werden können und auch sollten (vgl. Francis und Silvers 2007). Zusammenfassend ist also festzuhalten: Wenn die Person mit Demenz selbst rational wertvolle Projekte wertschätzt und die betreuenden Personen sie dabei unterstützen, das zu verfolgen, dann ist auch für Demenzpatienten ein sinnhaftes Leben möglich - ein Leben, für das gilt, dass subjektiv und rational Wertgeschätztes zusammentreffen.

Als Beleg mag auch hier wieder Richard Taylor herangezogen werden, der bis zu einem sehr fortgeschrittenen Stadium seiner Alzheimer-Erkrankung sein Projekt der Hilfe für andere Alzheimer-Patienten vorangetrieben hat und sich bewusst war, dass es dafür gute Gründe gibt:

What we do and learn about ourselves, each other, and life is just as important to share as what William Shakespeare or Emily Dickinson shared. We may not be able to express ourselves in three rhymed lines of five, seven, and five syllables (a haiku), but who, other than Miss Schetzel is counting? Hide under a bushel? No! I'm gonna let it shine. And you should, too! (Taylor 2015c, S. 187f.) 
Die Angst vor einem generellen Sinnverlust bei der Diagnosestellung „Demenz“ ist also in diesem Sinn nicht begründet, weil ein sinnhaftes Leben mit Demenz möglich ist. Allerdings kann diese Aussage nicht für alle Stadien der Demenz aufrechterhalten werden.

\section{Leben mit Demenz jenseits von Sinnhaftigkeit}

Ist die Demenzerkrankung in einem Stadium befindlich, in dem die kognitiven Fähigkeiten sehr stark eingeschränkt sind und die Fähigkeit verloren ist zu erkennen, was mit guten Gründen wertgeschätzt werden kann, dann ist ein im wolfschen Sinne sinnhaftes Leben nicht mehr möglich. Das mutet zunächst hart an, denn man möchte nicht sagen müssen, dass das Leben von Personen mit schwerster Demenz notwendiger Weise sinnlos wäre. An dieser Stelle muss man sich aber vor Augen führen, dass ,Sinnhaftigkeit“ hier in einer ganz bestimmten Weise zu verstehen ist. Jemand, der an einer schweren Form der Demenz erkrankt ist, kann zwar Zufriedenheit oder auch Glück empfinden, aber da für die subjektive Erfüllung ein kognitives Moment des Erkennens notwendig ist, dass das Angestrebte auch rationaler (bzw. objektiver) Weise wertvoll ist, ist Sinnhaftigkeit in diesem Fall verunmöglicht. Das, was subjektiv anziehend ist, und das, was rational wertvoll ist, ist in dem hybriden Ansatz von Wolf miteinander verknüpft. Ist das kognitive Element, das nach Wolf im Phänomen der Erfüllung verortet ist, nicht mehr vorhanden, so können subjektives und objektives Element nicht als miteinander verbunden angesehen werden. Das heißt nun nicht, dass im Leben der Person mit Demenz nicht Individualität verwirklicht werden könnte oder sich keine Zufriedenheit darin finden könnte. Es gilt immer noch, dass die Individualität und die Bedürfnisse zu respektieren sind. Was nicht möglich ist, ist ein in dem anspruchsvollen Sinn von Wolf sinnhaftes Leben. Würde man Erfüllung sparsamer - nur als etwas, das bloß subjektiv gut für jemanden ist verstehen, dann würde man den Wunsch nach einem sinnvollen Leben im wolfschen Sinn, den viele Menschen haben, nicht einfangen und vielmehr nach etwas anderem fragen (vgl. Wolf 2010b, S. 123).

Obwohl nun Personen mit Demenz vielleicht noch länger die Fähigkeit haben, etwas wertzuschätzen, wird die Fähigkeit zur reflexiven Einschätzung dieser Wertschätzung schon früher beeinträchtigt sein. Für die wolfsche Sinn-Konzeption ist es aber wichtig, dass eine bestimmte Beurteilung aus der Binnenperspektive desjenigen erfolgt, um dessen Leben es geht. Man muss nicht nur selbst etwas wertschätzen können, sondern auch fähig sein zu erkennen, ob man z. B. darauf auch stolz sein kann. Dafür muss derjenige, der etwas in seinem Leben wertschätzt, auch den bestimmten Schritt zurücktreten können, um zu beurteilen, ob das, was er wertschätzt, auch rational (bzw. objektiv) wertvoll ist. In Fällen der schweren Demenz wird das sicherlich nicht mehr der Fall sein.

Wenn aber die kognitiven Kapazitäten so eingeschränkt sind, dass die Person nicht mehr erkennen kann, was rational wertvoll ist, dann ist auch anzunehmen, dass sie diesen Aspekt des Lebens, um den es Wolf in ihrem Ansatz geht, nicht vermissen wird. Diese Person würde die Abwesenheit eines Projekts, das sowohl subjektiv als auch rational wertvoll ist, nicht bedauern. Aus der Sicht der Person mit Demenz würde ihr in diesem Sinne nichts fehlen. 


\section{Sollte man Angst vor dem Sinnverlust im Falle einer Demenzerkrankung haben?}

Die Angst vor dem Sinnverlust ist keine diffuse Angst, sondern sie ist auf etwas gerichtet. Sie ist intentional auf einen bestimmten Sachverhalt in der Zukunft ausgerichtet. Derjenige, der Angst empfindet, ist in einer bestimmten Art und Weise negativ eingestellt gegenüber dem, was er für bedrohlich hält. Ein Ziel dieses Aufsatzes war es, den Sachverhalt genauer zu fixieren, auf den die Angst gerichtet ist. Dazu wurde das Sinnverständnis Susan Wolfs herangezogen und als möglicher intentionaler Gehalt die Angst davor expliziert, kein Leben oder keine Lebensprojekte leben zu können, die zugleich subjektiv wie auch rational (bzw. objektiv) wertvoll sind.

Obwohl es nun schon fast auf der Hand liegt zu sagen, dass eine solche Angst gerechtfertigt ist, weil doch gezeigt wurde, dass jedenfalls im Endstadium der Erkrankung ein sinnhaftes Leben nicht möglich sein wird, wird ein solch allgemeines Urteil dem Phänomen in seiner Komplexität nicht gerecht. Für die weitere Ausführung ist es deshalb hilfreich, zwischen zwei möglichen Bezugspunkten der Angst zu unterscheiden: Erstens kann der Bezugspunkt sein, dass sich ein Sinn im Leben verändern wird, und zweitens, dass ein Sinn im Leben nicht mehr möglich sein wird.

Für den ersten Fall kann man noch einmal die Lebensgeschichte Richard Taylors illustrativ zu Rate ziehen. An der Person Taylors wurde gezeigt, dass man in seinem Fall sowohl im gesunden als auch im kranken Zustand anhand der wolfschen Kriterien sagen würde, dass sein Leben ein sinnvolles Leben ist. Allerdings hat sich das, was Taylors Leben zu einem sinnvollen Leben macht, verändert. Man kann vermuten, dass er sich vor seiner Erkrankung z. B. von seinem Leben als Psychologieprofessor erfüllt gefühlt hat, nach seiner Erkrankung aber hat sich das geändert. Sein späteres Leben ist das eines Alzheimer-Aktivisten und man kann vermuten, dass ihn auch dies erfüllt. Der Sinn im Leben Taylors hat eine Wandlung erfahren - so, wie sich auch die Persönlichkeit Taylors verändert hat. Es ist gut möglich, dass der gesunde Mensch das Projekt, das Erleben einer Demenzerkrankung zu dokumentieren, nicht wertschätzt, wohingegen er das im erkrankten Zustand tut.

Die Angst bei der Diagnosestellung kann sich also darauf beziehen, dass man dasjenige, was dem Leben im Falle der Demenzerkrankung Sinn geben könnte, nicht akzeptabel findet. Das impliziert, dass man erstens sein zukünftiges Selbst nicht mehr zu dem verpflichten kann, was man zurzeit wertschätzt. Zweitens ist aber bei der Beurteilung der Akzeptabilität zu berücksichtigen, dass sich auch die Persönlichkeit im Laufe der Zeit verändern wird. Man muss in Betracht ziehen, dass es der zukünftigen Person möglich ist, sich von entsprechenden Projekten erfüllt zu fühlen. Die Bewertung der Veränderung beinhaltet eine Beschreibung des zukünftigen Selbst, das etwas in seinem Leben wertschätzt: etwas, das subjektiv und rational (bzw. objektiv) wertvoll ist. Verkomplizierend kommt hinzu, dass es möglich ist, dass die spätere Person mit Demenz sich an ein früheres (kompetentes) Selbst - vermutlich in Teilen - erinnern kann und die Person mit Demenz möglicherweise etwas vermissen wird, das sie früher als kognitiv kompetente Person erfüllt hat.

Ein weiterer Bezugspunkt der Angst könnte sein, dass es der erkrankten Person im Spätstadium der Demenz, aufgrund kognitiver Einbußen, nicht mehr möglich 
sein wird, ein sinnhaftes Leben zu führen. Hier ist zu beachten, dass man in der Beurteilung der Veränderung seiner selbst berücksichtigt, dass in die Beschreibung seines späteren Selbst eingehen sollte, dass die spätere Person die Verunmöglichung, ein sinnhaftes Leben führen zu können, nicht bedauern wird.

Erst wenn man beide, zugegebenermaßen nicht leicht zu fassenden Elemente berücksichtigt, kann der/die erkrankte Person umfänglich beurteilen, ob man eine Veränderung seiner selbst in Bezug auf die Sinnhaftigkeit des Lebens bedrohlich und damit ablehnend befindet, oder ob man ihr indifferent gegenüberstehen kann oder sie sogar evtl. annehmen kann. Wie man diese Sachlage befindet, das ist allerdings individuell zu entscheiden. Ein Sinn im Leben ist je individuell verschieden, da Sinn in der hybriden Konstruktion Wolfs immer auch an das Subjekt gebunden ist. Für die mögliche Bewältigung der Angst scheint hier wichtig zu sein, dass verschiedene Strategien des Umgangs mit Ängsten als aussichtsreich befunden werden, und die gilt es an die Situation der Erkrankten anzupassen (Bjørkløf et al. 2019).

\section{Zusammenfassung}

Die Beurteilung der Angst vor dem Sinnverlust ist kompliziert, da sich die Persönlichkeit über die Zeit hinweg verändert. Die Persönlichkeit der Person, die die Diagnose gestellt bekommt, ist eine andere als zum Zeitpunkt der manifesten Demenz. Diese Veränderung der Persönlichkeit sollte in die Bewertung der Angst miteingehen. Hier wurde dafür argumentiert, dass man unter Rückgriff auf Überlegungen von Jaworska davon ausgehen kann, dass es Personen bis zu einer mittelschweren Demenz möglich ist, ein sinnvolles Leben zu leben. Ab dem Stadium der schweren Demenz, bei dem die kognitiven Einbußen so groß sind, dass ein reflexiver Schritt zurück nicht mehr möglich ist, ist allerdings ein sinnhaftes Leben nicht mehr möglich. In diesen Fällen vermisst die Person mit schwerster Demenz ein Fehlen von Sinnhaftigkeit jedoch nicht mehr. Ob die Angst vor einem Sinnverlust gerechtfertigt ist, hat etwas damit zu tun, wie man von der Perspektive der Diagnosestellung aus eine Beschreibung seiner selbst im Zustand der Demenz bewertet: einer Person, für die es eine ganze Zeit mit entsprechender Hilfe noch möglich ist, ein sinnhaftes - wenn auch wahrscheinlich auf eine andere Art sinnhaftes - Leben zu führen und für die es in der Endphase der Demenz zwar noch möglich ist, ein zufriedenes, aber kein sinnhaftes Leben mehr zu führen. Sie wird Letzteres aber auch nicht bedauern.

Funding Open Access funding enabled and organized by Projekt DEAL.

Open Access Dieser Artikel wird unter der Creative Commons Namensnennung 4.0 International Lizenz veröffentlicht, welche die Nutzung, Vervielfältigung, Bearbeitung, Verbreitung und Wiedergabe in jeglichem Medium und Format erlaubt, sofern Sie den/die ursprünglichen Autor(en) und die Quelle ordnungsgemäß nennen, einen Link zur Creative Commons Lizenz beifügen und angeben, ob Änderungen vorgenommen wurden.

Die in diesem Artikel enthaltenen Bilder und sonstiges Drittmaterial unterliegen ebenfalls der genannten Creative Commons Lizenz, sofern sich aus der Abbildungslegende nichts anderes ergibt. Sofern das betreffende Material nicht unter der genannten Creative Commons Lizenz steht und die betreffende Handlung nicht nach gesetzlichen Vorschriften erlaubt ist, ist für die oben aufgeführten Weiterverwendungen des Materials die Einwilligung des jeweiligen Rechteinhabers einzuholen. 
Weitere Details zur Lizenz entnehmen Sie bitte der Lizenzinformation auf http://creativecommons.org/ licenses/by/4.0/deed.de.

\section{Einhaltung ethischer Richtlinien}

Interessenkonflikt S. Hiekel gibt an, dass kein Interessenkonflikt besteht.

Ethische Standards Für diesen Beitrag wurden von der Autorin keine Studien an Menschen oder Tieren durchgeführt. Für die aufgeführten Studien gelten die jeweils dort angegebenen ethischen Richtlinien.

\section{Literatur}

Bender MP, Cheston R (1997) Inhabitants of a lost kingdom: a model of the subjective experiences of dementia. Ageing Soc 17:513-532

Bjørkløf G, Helvik A-S, Ibsen TL, Telenius EW, Grov EK, Eriksen S (2019) Balancing the struggle to live with dementia: a systematic meta-synthesis of coping. BMC Geriatr 19:1-24

Cheston R, Christopher G, Ismail S (2015) Dementia as an existential threat: the importance of self-esteem, social connectedness and meaning in life. Sci Prog 98(4):416-419

Clark A, Chalmers D (1998) The extended mind. Analysis 58(1):7-19

Dewitte L, van Wijngaarden E, Schellekens T, Vandenbulcke M, Dezutter J (2021) Continuing to participate in the dance of life as oneself: the lived experiences of meaning in life for older adults with Alzheimer's disease. GERONT. https://doi.org/10.1093/geront/gnaa206

Diehl U (2009) Human suffering as a challenge for the meaning of life. Existenz. Int J Philos Religion Polit Arts 4(2):36-44

Dworkin R (1993a) Life past reason. In: Dworkin R. Life's dominion. Random House, New York, S 218-237

Dworkin R (1993b) Dying and living. In: Dworkin R. Life's dominion. Random House, New York, S $179-217$

Francis LP, Silvers A (2007) Liberalism and individually scripted theories of the good. Meeting the challenge of dependant agency. Soc Theory Pract 33(2):311-334

Halbig C (2018) Sinn - eine dritte Dimension guten Lebens? Z Prakt Philos 5(2):55-78

Hallich O (2011) Selbstbindungen und medizinischer Paternalismus: Zum normativen Status von Odysseus-Anweisungen. Z Philosoph Forsch 65(2):151-172

Hallich O (2019) Zwei Arten von Selbstbindungen. Z Ethik Moralphilos 2:305-314

Hiekel S (2018) Fitting fulfilment-Fitting objective or rational attractiveness? Z Ethik Moralphilos 1(1):57-74

Hiekel S (2020) Was gibt dem Leben einen Sinn? Susan Wolf. In: Müller-Salo J (Hrsg) Analytische Philosophie. Eine Einführung in 16 Fragen und Antworten. utb, Paderborn, S 173-188

Holst G, Hallberg IR (2003) Exploring the meaning of everyday life, for those suffering from dementia. Am J Alzheimers Dis Other Dement 18(6):359-365

Jaworska A (1997) Rescuing Oblomov. A search for convincing justifications of value. Dissertation. Harvard University,

Jaworska A (1999) Respecting the margins of agency: Alzheimer's patients and the capacity to value. Philos Public Aff 28(2):105-138

Johannessen A, Möller A (2011) Experiences of persons with early-onset dementia in everyday life: a qualitative study. Dementia 12(4):410-424

Kipke R (2018) Der Sinn des Lebens und das gute Leben. Z Prakt Philos 5(2):180-202

Kipke R, Rüther M (2021) Die Sinnfrage in der Medizinethik. Editorial. Ethik Med: (im Erscheinen)

Koethe J (2010) Comment. In: Wolf S. Meaning in life and why it matters. Princeton University Press, Princeton, S 67-74

Korsgaard C (2014) The sources of normativity, 20. Aufl. Cambridge University Press, Cambridge

Korsgaard C (2018) What's different about being human? In: Korsgaard C. Fellow creatures-Our obligations to the other animals. Oxford University Press, Oxford, S 36-52

Lakotta B (2010) Ein Leben wie im Fegefeuer. https://www.spiegel.de/spiegel/print/d-69277670.html. Zugegriffen: 26. Mai 2020

Lesser AH (2006) Dementia and personal identity. In: Hughes JH, Louw SJ, Sabat SR (Hrsg) Dementia-mind, meaning and the person. Oxford University Press, Oxford, S 55-61 
Mackenzie C, Stoljar N (2000) Autonomy refigured. In: Mackenzie C, Stoljar N (Hrsg) Relational autonomy. Feminist perspectives on autonomy, agency, and the social self. Oxford University Press, Oxford

Mackie JL (1977) Ethics. Inventing right and wrong. Penguin Books, Harmondsworth

McMillan J (2006) Identity, self, and dementia. In: Hughes JH, Louw SJ, Sabat SR (Hrsg) Dementia - mind, meaning and the person. Oxford University Press, Oxford, S 63-70

Muders S (2018) Die Eigenständigkeit des sinnvollen Lebens innerhalb des guten Lebens. Z Prakt Philos 5(2):79-118

O'Connor D, Phinney A, Smith A, Small J, Purvers B, Perry J, Drance E, Donelly M, Chaudhury H, Beattie L (2007) Personhood in dementia care. Developing a research agenda for broadening the vision. Dementia 6(1):121-142

Quante M (2007) Person. De Gruyter, Berlin, New York

Quante M (2013) Personale Identität und tiefe Hirnstimulation. In: Manzeschke A, Zichy M (Hrsg) Therapie und Person. Ethische und anthropologische Aspekte der tiefen Hirnstimulation. Mentis, Münster

Radden J, Fordyce JM (2006) Into the darkness: losing identity with dementia. In: Hughes JH, Louw SJ, Sabat SR (Hrsg) Dementia—mind, meaning and the person. Oxford University Press, Oxford, S 71-88

Rojek T (2020) Person und Persönlichkeit. In: Quante M, Goto H, Rojek T, Segawa S (Hrsg) Der Begriff der Person in systematischer und historischer Perspektive. Ein deutsch-japanischer Dialog. Mentis, Paderborn, S 145-160

Rüther M (2021a) Meaning in Life oder: Die Debatte um das sinnvolle Leben - Überblick über ein neues Forschungsthema in der analytischen Ethik: Teil 1: Grundlagen. Z Philos Forsch 75(1):115-155

Rüther M (2021b) Meaning in Life oder: Die Debatte um das sinnvolle Leben - Überblick über ein neues Forschungsthema in der analytischen Ethik: Teil 2: normative und inhaltliche Fragen. Z Philos Forsch 75(2):316-354

Rüther M, Muders S (2014) Die Frage nach dem Sinn des Lebens in der gegenwärtigen Philosophie. Z Philos Forsch 68(1):96-122

Schöne-Seifert B, Stier M, Reichardt JO (2019) Zur moralischen Autorität von Odysseus-Verfügungen: Definitionen, Unterscheidungen, Rechtfertigungen. Z Ethik Moralphilos 2:315-329

Taylor R (2015a) What is it like to have Alzheimer's disease-Three years later. In: Taylor R (ed) Alzheimer's from the inside out, 5. Aufl. HPP, Baltimore, London, Sydney, S 35-36

Taylor R (2015b) Falling. In: Taylor R (ed) Alzheimer's from the inside out, 5. Aufl. HPP, Baltimore, London, Sydney, S 130-131

Taylor R (2015c) This little light of mine. In: Taylor R (ed) Alzheimer's from the inside out, 5. Aufl. HPP, Baltimore, London, Sydney, S 186-188

Wolf S (1997) Meaningful lives in a meaningless world. Quaestiones Infin 19:1-22

Wolf S (2010a) Meaning in life. In: Wolf S. Meaning in life and why it matters. Princeton University Press, Princeton, New Jersey, S 1-33

Wolf S (2010b) Response. In: Wolf S. Meaning in life and why it matters. Princeton University Press, Princeton, New Jersey, S 102-132

Wolf S (2010c) Meaning in life and why it matters. Princeton University Press, Princeton, New Jersey

Wolf S (2015) Meaning of lives. In: Wolf S. Varieties of values. Oxford University Press, Oxford, S 89-106

Wolf S (2016) Meaningfulness: a third dimension of the good life. Found Sci 21(2):253-269 